\title{
THE ASYMPTOTIC GIANT BRANCH
}

\author{
J. C. LATTANZIO AND C. A. FROST \\ Department of Mathematics, Monash University, Australia
}

\section{Introduction}

For stars with masses between about 1 and $8 M_{\odot}$ the ascent of the Asymptotic Giant Branch (AGB) is the last nuclear powered evolutionary stage. Following this the stars eject a planetary nebula and fade as white dwarfs. Although only short in duration, the AGB is very important due to the nucleosynthesis which occurs. Here we briefly review the species produced and the mechanism for their production. We then discuss the current uncertainties in quantitative predictions of this nucleosynthesis, which centre on our determination of convective boundaries.

\section{AGB Evolution}

The details of AGB evolution have been the subject of much theoretical work, and are, in one sense, quite well understood. The reader is referred to Iben \& Renzini (1983), Frost \& Lattanzio (1996a) and Lattanzio et al.(1996) for details. Briefly, an AGB star has the structure shown in Figure 1. The C-O core is the result of He burning, and will become the final white-dwarf remnant. Just above this is the He-shell. This is thermally unstable, and burns vigorously during shell flashes (or thermal pulses) but is essentially extinguished between them. Above the He-shell is the intershell region, so-called because it is between the $\mathrm{He}$ and $\mathrm{H}$-shells. Above the $\mathrm{H}$-shell is the convective envelope. During a thermal pulse, the He-shell will deliver some $10^{7} L_{\odot}$ for a brief period, and this enormous energy production results in the formation of a convective zone. This "flash-driven intershell convection" extends over the region shown in Figure 1, and thus mixes the products of (partial) helium burning throughout this region. The approximate composition of this zone is $25 \%$ carbon and $75 \%$ helium. Following the pulse, the helium luminosity decreases and the star expands. This essentially extinguishes the $\mathrm{H}$-shell,

T.R. Bedding et al. (eds.),

Fundamental Stellar Properties: The Interaction between Observation and Theory, 373-380.

(C) 1997 IAU. Printed in the Netherlands. 


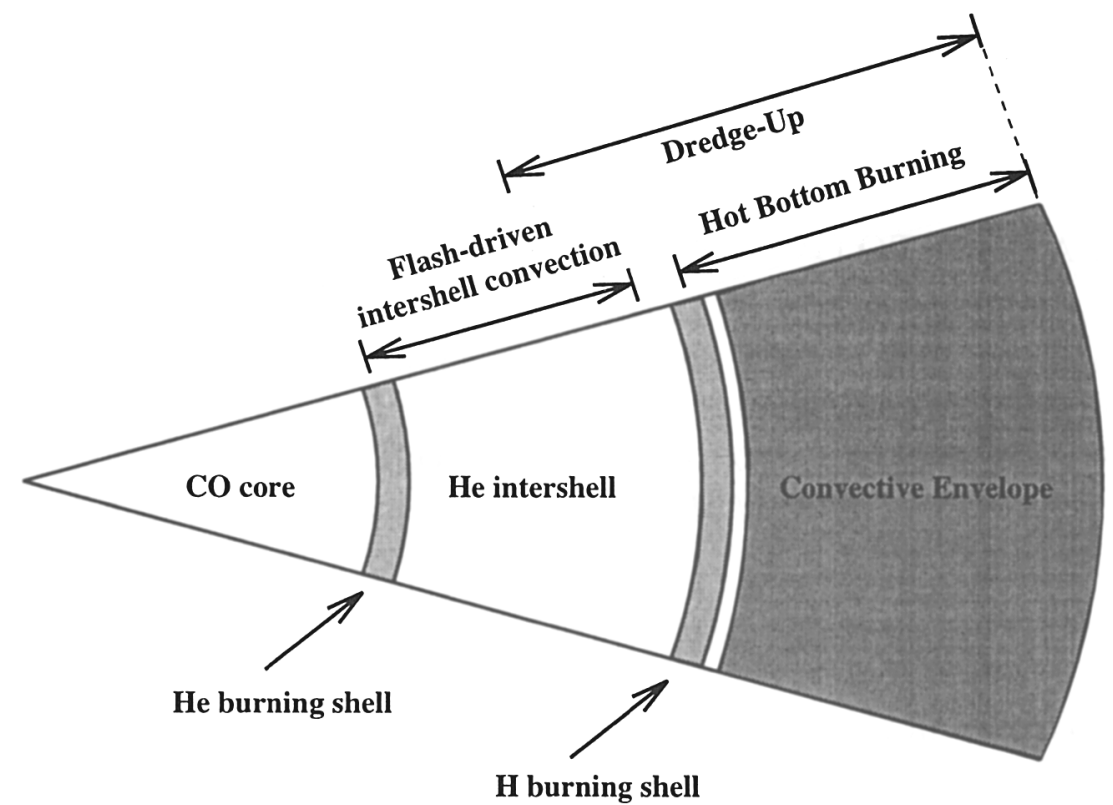

Figure 1. Schematic structure of an AGB star. During a thermal pulse the intershell convection extends over the region marked by "flash-driven intershell convection". During the dredge-up phase, the convective envelope moves inward to the depth marked by "dredge-up". For massive stars, during the interpulse phase the convective envelope penetrates the top of the H-shell, so that envelope convection extends down to the region marked "hot bottom burning".

and the bottom of the convective envelope moves inwards in mass. After a small number of pulses, this convection penetrates the maximum outward extent of the flash-driven convective zone and this results in the mixing of freshly produced carbon to the stellar surface. As the star begins to contract back to its normal configuration, the H-shell is re-ignited and provides all of the energy during the next interpulse phase, until the following pulse.

For more massive stars (above about $4 M_{\odot}$ ) the bottom of the convective envelope penetrates into the top of the H-shell, and some nuclear reactions take place at the bottom of the convective envelope. This is known as "hot bottom burning" (hereafter HBB), and is shown schematically in Figure 1. The termination of the AGB phase is controlled by the mass loss. When the envelope becomes sufficiently thin, the star leaves the AGB. In the calculations presented below we used the formula of Vassilliadis \& Wood (1993).

\section{AGB Nucleosynthesis}

\subsection{NEUTRON-CAPTURE}

The production of post-iron elements in AGB stars is well known, both observationally and theoretically. We now believe that the neutron source 


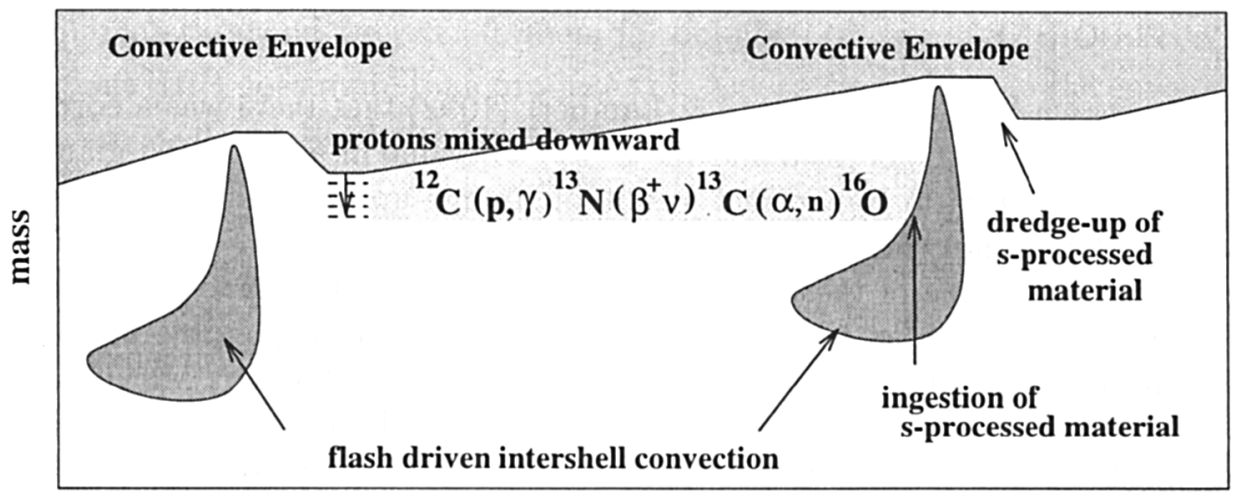

time

Figure 2. Schematic structure of two consecutive thermal pulses, showing how the downward mixing of hydrogen leads to the production of ${ }^{13} \mathrm{C}$ and then $s$-processing. Note that this $s$-processed material is later dredged to the surface of the star.

active in these stars (at least the lower masses) is ${ }^{13} \mathrm{C}$. But how exactly does this ${ }^{13} \mathrm{C}$ arise? This remains a serious problem for the models. While it is true that some ${ }^{13} \mathrm{C}$ is produced by $\mathrm{CNO}$ cycling in the $\mathrm{H}$-shell, this is nowhere near enough to produce the neutron exposures inferred from observations of these stars. We will return to this problem later, but for now we will assume that some kind of extra-mixing takes place at the bottom of the convective envelope during the dredge-up phase. This has indeed been found by Iben \& Renzini (1982a,b), but has not been reproduced consistently. If this mixing occurs, then small amounts of hydrogen are mixed into a region which is relatively rich in ${ }^{12} \mathrm{C}$. During the subsequent interpulse phase, these regions heat and the protons are captured by the ${ }^{12} \mathrm{C}$ to produce ${ }^{13} \mathrm{C}$. It is crucial that there is not too much hydrogen in this region, or the $\mathrm{CN}$ cycle will progress further to ${ }^{14} \mathrm{~N}$, destroying the all-important ${ }^{13} \mathrm{C}$. This ${ }^{~}{ }^{13} \mathrm{C}$-pocket" was believed to sit in the star and wait until the next thermal pulse, when it would be engulfed by the intershell convection. The high temperatures present would then release the neutrons and the $s$-processing would occur in the convective intershell region. But it has been shown recently (Straniero et al.1995; Lattanzio et al.1996; Mowlavi et al.1996) that the temperatures in the intershell are sufficiently high, and/or the interpulse duration sufficiently long, that the ${ }^{13} \mathrm{C}$ burns through to ${ }^{16} \mathrm{O}$, thus releasing the neutrons in situ where the $s$-processing will then take place. Later, when this region is mixed into the flash-driven convection zone, the results of the neutron captures are also mixed into the convective zone. This situation is shown schematically in Figure 2. The details of the $s$-processing are not our concern here: the interested reader is referred to Gallino \& Busso (1997). 


\subsection{FLUORINE}

It was shown by Jorissen, Smith \& Lambert (1992) that there was a correlation between the $\mathrm{C} / \mathrm{O}$ ratio and the $\left[{ }^{19} \mathrm{~F} /{ }^{16} \mathrm{O}\right]$ value in galactic red-giants. Since the $\mathrm{C} / \mathrm{O}$ ratio is a direct result of dredge-up (at least for masses where $\mathrm{HBB}$ is not acting) this implicates shell-flashes as a source of ${ }^{19} \mathrm{~F}$ enhancement. Some of these enhancements are quite large, reaching values of $\left[{ }^{19} \mathrm{~F} /{ }^{16} \mathrm{O}\right]$ as high as 1.5 . Forestini et al.(1992) outlined possible production mechanisms for this ${ }^{19} \mathrm{~F}$. These ideas were further refined by Mowlavi et al.(1996). The basic ideas behind the production of ${ }^{19} \mathrm{~F}$ are: we begin interior to the $\mathrm{H}$-shell, in the region consisting mostly of ${ }^{4} \mathrm{He}$ with some ${ }^{14} \mathrm{~N}$ (from CNO cycling). ${ }^{14} \mathrm{~N}$ captures an alpha particle to produce ${ }^{18} \mathrm{~F}$ which then beta decays to ${ }^{18} \mathrm{O}$. Assuming a source of protons (see below!) ${ }^{18} \mathrm{O}$ then produces ${ }^{15} \mathrm{~N}$ via ${ }^{18} \mathrm{O}(p, \alpha){ }^{15} \mathrm{~N}$. This ${ }^{15} \mathrm{~N}$ can then capture another ${ }^{4} \mathrm{He}$ nucleus to produce ${ }^{19} \mathrm{~F}$. But we need a proton source for this chain of reactions to take place. There are two likely sources of protons. ${ }^{13} \mathrm{C}$ suffers $(\alpha, n)$ reactions to produce ${ }^{16} \mathrm{O}$ and neutrons. These neutrons are used to produce the enhancements in $s$-process elements seen in these stars, but they are also available for ${ }^{14} \mathrm{~N}$ and ${ }^{26} \mathrm{Al}$ to capture. Each undergoes $(n, p)$ reactions and hence produces protons. The details of this process depend primarily on the temperature of the intershell region, both during a pulse and during the interpulse phase. The temperature, of course, also depends on the metallicity of the star as well as the core-mass, and details are provided in Mowlavi et al.(1996). An important point for our purposes is that once ${ }^{19} \mathrm{~F}$ is dredged to the surface, it is destroyed by any $\mathrm{HBB}$ present via ${ }^{19} \mathrm{~F}(p, \alpha){ }^{16} \mathrm{O}$. Thus stars with enhanced ${ }^{19} \mathrm{~F}$ are presumably those of lower masses, where HBB does not take place. For further details see Mowlavi et al.(1996). Finally, we note that to produce the largest observed enhancements of ${ }^{19} \mathrm{~F}$ seems to require a substantial source of ${ }^{13} \mathrm{C}$, just as is needed for the $s$-process abundances. The exact mechanism responsible for producing this ${ }^{13} \mathrm{C}$ remains unknown (see below).

\subsection{HOT BOTTOM BURNING}

The maximum envelope temperature for a $6 M_{\odot}$ model with $Z=0.02$ was found to rise with each pulses to a peak of $70 \times 10^{6} \mathrm{~K}$. Toward the end of the evolution mass-loss results in a decreasing envelope mass, and hence the temperature at the base of the envelope decreases, and HBB is terminated just before the star leaves the AGB. This HBB burns $\mathrm{H}$ via the $\mathrm{CNO}, \mathrm{Ne}-\mathrm{Na}$ and $\mathrm{Mg}-\mathrm{Al}$ cycles. But the first thing to happen is a significant increase in the ${ }^{7} \mathrm{Li}$ content of the stellar envelope. Cameron \& Fowler (1971) suggested a mechanism for the production of ${ }^{7} \mathrm{Li}$ which required HBB. Boothroyd \& Sackmann (1992) showed quantitatively that such a scenario can work in the 
required stars, and we refer to them for details.

But HBB does more than produce ${ }^{7} \mathrm{Li}$. Any carbon added to the envelope by the repeated third dredge-up is converted into both ${ }^{13} \mathrm{C}$ and ${ }^{14} \mathrm{~N}$. In fact, the timescales are such that the entire envelope is mixed through this high-temperature region many times, and the resulting ratio of ${ }^{12} \mathrm{C} /{ }^{13} \mathrm{C}$ is about 3.5, appropriate to the equilibrium operation of the CN cycle. Further processing burns the ${ }^{13} \mathrm{C}$ into ${ }^{14} \mathrm{~N}$, which is able to prevent the star from becoming a Carbon star (see also Wood et al.1983, Boothroyd et al.1993).

A detailed analysis of the nucleosynthesis occurring during $\mathrm{HBB}$ is not the intention of this paper, but we mention here some of the more noteworthy changes. Firstly there is efficient destruction of the ${ }^{19} \mathrm{~F}$ produced by thermal pulses! Hence, as stated earlier, we would not expect to see relatively massive stars with enhanced ${ }^{19} \mathrm{~F}$. Secondly, there is a dramatic destruction of ${ }^{18} \mathrm{O}$ as first discussed by Boothroyd et al.(1995). Finally, we also see a production of ${ }^{26} \mathrm{Al}$ from the $\mathrm{Mg}-\mathrm{Al}$ cycle. This is even more pronounced in models of lower metallicity (Lattanzio et al.1997a,b). This is very important for the interpretation of the isotopic ratios measured in grains recovered from meteorites (see below).

\section{New "Observations": Meteorite Grains}

We have recently seen the exploitation of a new source of information about the composition of stellar material, provided by measurements of isotopic ratios in individual grains found in meteorites. A significant advantage of these measurements is that they can provide information about many elements for each grain, and since each grain has condensed in the outflow from a single star, we obtain much compositional information from a single stellar source.

The grains of interest to us are the silicon carbide $(\mathrm{SiC})$ and oxide grains, especially corundum $\left(\mathrm{Al}_{2} \mathrm{O}_{3}\right)$. Because the $\mathrm{SiC}$ grains must form in a carbonrich environment it is believed that these grains originated in the envelopes of Carbon stars. The interest in corundum these is due to the oxygen and aluminium isotopic ratios, which show evidence of the three dredge-up episodes as well as HBB. It appears that a satisfactory explanation of all these grains requires us to consider stars of varying initial masses and a spread in the initial oxygen isotopic ratios, as discussed by Boothroyd et al. (1994). The effect of HBB on oxygen isotopic ratios has been calculated by Boothroyd et al. (1995), with particular reference to meteoritic data. Many of the oxide grains of Nittler et al. (1994) have been analysed for Al-Mg and show excesses of ${ }^{26} \mathrm{Mg}$ but have normal ${ }^{25} \mathrm{Mg} /{ }^{24} \mathrm{Mg}$. This indicates that live ${ }^{26} \mathrm{Al}$ has decayed in situ to produce the ${ }^{26} \mathrm{Mg}$. The inferred initial ${ }^{26} \mathrm{Al} /{ }^{27} \mathrm{Al}$ ratios are as large as 0.016 , quite consistent with the calculations reported in Lattanzio et al. $(1997 \mathrm{a}, \mathrm{b})$. There is also a wealth of data available for other species 


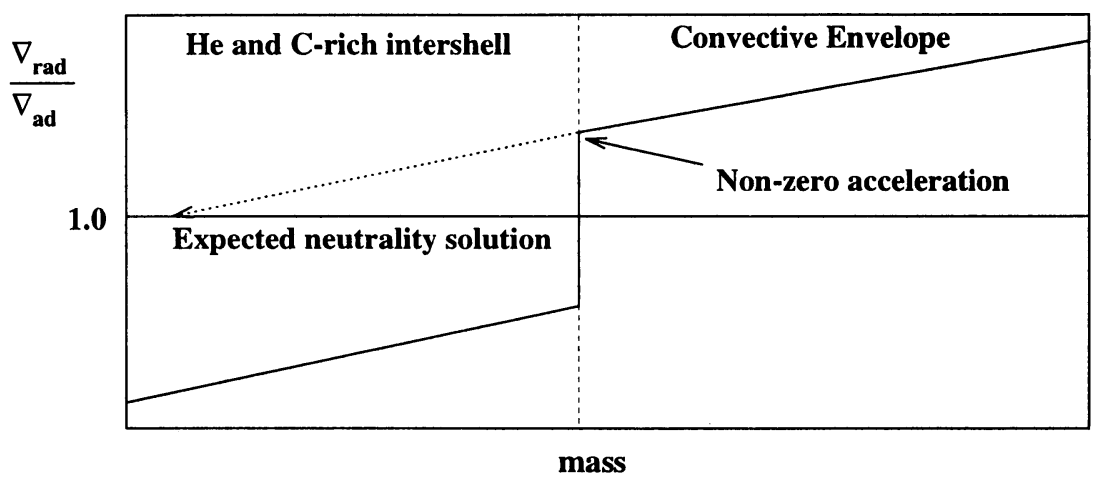

Figure 3. Schematic diagram showing the behaviour of the important temperature gradients at the bottom of the convective envelope during dredge-up in intermediate mass stars.

(see, for example, Gallino et al. 1994) but this will not be addressed here, except to remind us that we ignore this new source of highly accurate and wonderful data at our peril.

\section{Model Limitations}

Synthetic evolutionary calculations (e.g. Groenewegen \& deJong 1993) using the best theoretical estimates for AGB evolution are unable to match the observed LMC Carbon star distribution. The observations require dredgeup to begin earlier in the evolution (i.e. at lower values of the core-mass) and to penetrate deeper into the star than current models predict. Both of these problems are related to determining the edge of a convective zone under complicated physical conditions.

There is, of course, a composition discontinuity at the bottom of the convective envelope during dredge-up: in the envelope the material is $\mathrm{H}$ rich, but the envelope is growing into material which was previously part of the flash-driven intershell convective zone, and hence is about $75 \% \mathrm{He}$ and about $25 \% \mathrm{C}$ (see Figure 3). The Schwarzschild criterion for determining the convective boundary relies on finding the position where the acceleration is zero, which is where $\nabla_{r a d}=\nabla_{a d}$. Convective eddies will still have a non-zero momentum when they reach this boundary, and hence they will penetrate into the radiatively stable region where they are decelerated to zero velocity. This is what is usually referred to in the literature as "convective overshoot". But for third dredge-up, the boundary is even more prone to extra-mixing, since the acceleration (as well as the momentum) is non-zero at the convective boundary. Exactly how the star will mix, to achieve the expected convective neutrality, is uncertain. It is, of course, a hydrodynamical problem. Somehow we expect the convective region to grow into the intershell region, until the 


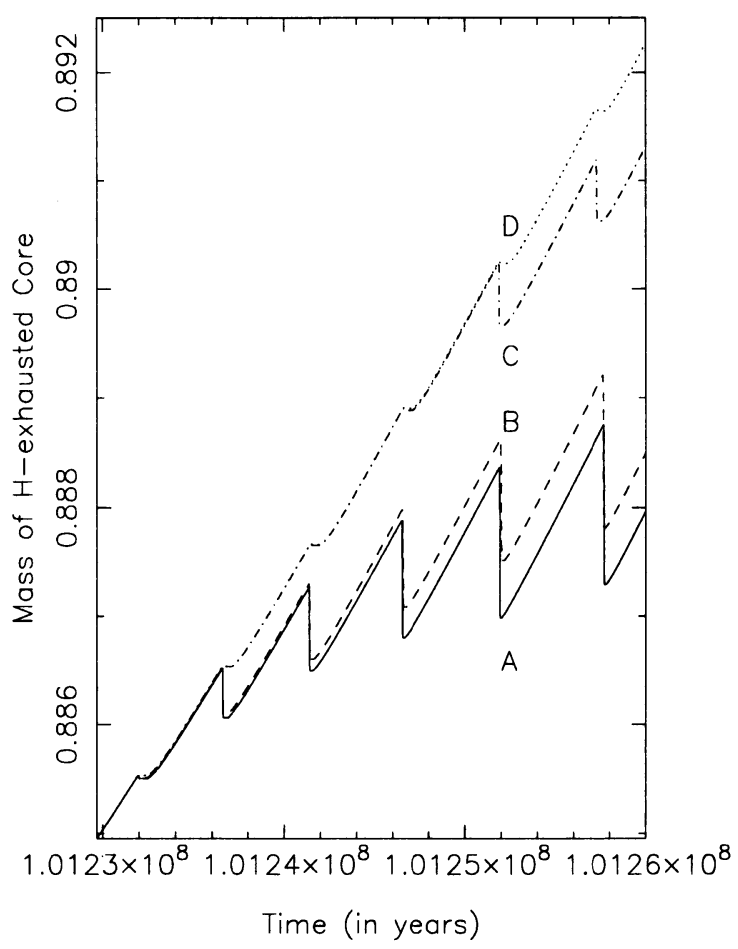

Figure 4. Four different algorithms for mixing, and four different amounts of dredge-up. See Frost \& Lattanzio (1996b) for details.

gradients smoothly approach each other (see the dotted line in Figure 3). From this configuration we would still expect the usual overshoot.

This situation has been investigated in some detail by Frost \& Lattanzio (1996b) who found that the depth of dredge-up depended critically on assumptions made at the boundary of the convective region, as well as the way in which the mixing was handled within the evolutionary calculation (eg. if the mixing is performed after each iteration, or only after a model has converged). For example, Figure 4 shows the mass of the $\mathrm{H}$-exhausted core as a function of time for a $5 M_{\odot}$ model with $Z=0.004$. Each of the four evolutionary sequences A, B, C and D began with the same model; each uses a somewhat different algorithm for dealing with the convective boundary and associated mixing. The effect on dredge-up is dramatic, varying from none in case D to very deep in case A (see Frost \& Lattanzio 1996b for details). Clearly this situation is not satisfactory, and much theoretical work must be done to clarify this picture.

Current work in progress has shown that the depth of dredge-up dramatically alters the evolution of the star. Deep dredge-up cools the intershell 
region, and slows the advance of the He-shell to almost zero. Thus we find almost stationary shell burning. The depth of the dredge-up depends also on the treatment of the entropy cost of mixing dense material upward in a gravitational field (Wood 1981). We have much to learn about this complicated phase of evolution, and work is continuing.

\section{Conclusion}

We have attempted to explain how AGB stars make many of the interesting nuclear products seen in their spectra. We have also pointed out where the models are most in doubt: the determination of convective boundaries. A significant quantitative advance in our understanding of nucleosynthesis in AGB stars requires us to tackle the difficult problem of hydrodynamic turbulent convection in three dimensions. Are there any volunteers?

\section{References}

Boothroyd, A. I. \& Sackmann, I.-J., 1992, Astrophys. J. Lett, 393, L21.

Boothroyd, A. I., Sackmann, I.-J., \& Ahern, S. C., 1993, Astrophys. J., 416, 762.

Boothroyd, A. I., Sackmann, I.-J., \& Wasserburg, G. J., 1994, Astrophys. J. Lett, 430, L77.

Boothroyd, A. I., Sackmann, I.-J., \& Wasserburg, G. J., 1995, Astrophys. J. Lett, 442, L21.

Cameron, A. G. W., \& Fowler, W. A., 1971, Astrophys. J., 164, 111.

Forestini, M., Goriely, S., Jorissen, \& Arnould, M., 1992, Astron. Astrophys., 261, 157.

Frost, C.A. \& Lattanzio, J. C., 1996a in Stellar Evolution: What Should Be Done?, Proceedings of the 32nd Liege Colloquium, Eds A. Noels et al, 307.

Frost, C. A $i$, \& Lattanzio, J. C., 1996b, Astrophys. J., 473, 383.

Gallino, R., et al.1994, Astrophys. J., 430 , 858.

Gallino, R. \& Busso, M., 1997, in Proceedings of Astrophysical Implications of Laboratory Study of Interstellar Grains, in press.

Groenewegen, M. A. T., \& de Jong, T., 1993, Astron. Astrophys., 267, 410.

Iben, I., Jr., \& Renzini, A., 1982a, Astrophys. J. Lett, 259, L791.

Iben, I., Jr., \& Renzini, A., 1982a, Astrophys. J. Lett, 263, L231.

Iben, I., Jr., \& Renzini, A., 1983, Ann. Rev. Astr. Ap., 21, 271.

Jorissen, A., Smith, V. V., \& Lambert, D. L., 1992, Astron. Astrophys., 261, 164.

Lattanzio, J. C., Frost, C. A., Cannon, R. C., \& Wood, P. R., 1996, Mem. Astro. Soc. Italia, in press.

Lattanzio, J. C., Frost, C. A., Cannon, R. C., \& Wood, P. R., 1997a, "Nucleosynthesis in Intermediate Mass Stars" in The Carbon Star Phenomenon, Proceedings of IAU Symposium 177, Ed R. F. Wing, in press.

Lattanzio, J. C., Frost, C. A., Cannon, R. C., \& Wood, P. R., 1997b, in preparation.

Mowlavi, N., Jorissen, A., \& Arnould, M., 1996, Astron. Astrophys., 311, 803.

Nittler, L. R., Alexander, C. M. O'D., Gao, X., Walker, R., \& Zinner, E. K., 1994, Nature, $370,443$.

Straniero, O., Gallino, R., Busso, M., Chieffi, A., Limongi, M., and Salaris, M., 1995, Astrophys. J. Lett, 440, L85.

Vassilliadis, E. \& Wood, P. R., 1993, Astrophys. J., 413, 641.

Wood, P. R., 1981, Astrophys. J., 248, 311.

Wood, P. R., Bessell, M. S., \& Fox, M. W., 1983, Astrophys. J., 272, 99. 